\title{
Tinea Capitis in Adults
}

\author{
Ornella Cervetti, Paola Albini, Veronica Arese, Federica Ibba, \\ Manuela Novarino, Michele Panzone \\ Clinica Dermatologica Torino, Torino, Italy \\ Email: ornella.cervetti@unito.it
}

Received October 15, 2013; revised November 15, 2013; accepted November 22, 2013

Copyright (C) 2014 Ornella Cervetti et al. This is an open access article distributed under the Creative Commons Attribution License, which permits unrestricted use, distribution, and reproduction in any medium, provided the original work is properly cited. In accordance of the Creative Commons Attribution License all Copyrights (C) 2014 are reserved for SCIRP and the owner of the intellectual property Ornella Cervetti et al. All Copyright (C) 2014 are guarded by law and by SCIRP as a guardian.

\begin{abstract}
Objective: To determine the epidemiology and the aetiology of tinea capitis in the elderly in Turin (Italy). Methods: We prospectively collected all cases of adult tinea capitis in the Dermatology Clinic of the University of Turin from January 1997 to December 2012. Results: 13 patients (4 males and 9 females) with a mean age of 56.5 years were found to be affected by tinea capitis among a total number of $508(2.6 \%)$. Diagnosis was made on clinical appearance and mycological examination. Culture identified M. canis in 7 patients (53.8\%), T. mentagrophytes in 3 patients (23.1\%), T. rubrum in 2 patients (15.4\%) and T. violaceum in 1 patient (7.7\%). 6 cases reported contact with animals. Treatment consisted in administration of Griseofulvin at the dose of $500 \mathrm{mg} / \mathrm{d} \mathrm{or}$ Terbinafine at the dose of $250 \mathrm{mg} / \mathrm{d}$ for at least 8 weeks. A complete recovery was observed in 10 patients, whereas 3 of them suffered from cicatritial alopecia. Conclusion: This study wants to highlight the importance of considering tinea capitis as a differential diagnosis in elderly patients with scaly scalp lesions, even though it is considered to be rare in adults.
\end{abstract}

\section{KEYWORDS}

Dermatophytosis; Tinea Capitis; Scalp Ringworm; Adults; Elderly

\section{Introduction}

The dermatophytes use keratin to grow; therefore all keratin-containing body parts can become infected [1].

Tinea capitis, or scalp ringworm, is the most common dermatophyte infection of the scalp [2], and it is estimated to represent $1 \%$ of all superficial fungal infections in northern and Western Europe [3].

The epidemiology of tinea capitis varies within different geographical areas in the world [2]; in Europe, the most frequent aetiological agents are $M$. canis [4], T. verrucosum (zoophilic species), T. tonsurans, T. violaceum and T. sudanense (anthropophilic species) [5]. Contaminations by some of these species are increasing because of immigration (e.g. T. violaceum) [3].

Literature reports that in Europe, the incidence of $M$. canis infections has considerably increased during the recent past years [5]. This species, which is now the main causative agent of tinea capitis in Italy [6,7], is very commonly found in cats and dogs, causing in these animals inapparent and lifelong infections. Without its ani- mal reservoir, $M$. canis cannot spread in the human population [3,8]; human-to-human transmission is not frequent and it is described as self-limiting [9].

Moreover, we find in literature an increase in anthropophilic infections, mainly in urban areas [3].

The increasing incidence and the continuous changes of the predominant aetiological agents of tinea capitis make necessary and constant update about this subject.

Tinea capitis mainly affects children and rarely adults [3,5] (less than $3 \%$ of all reported cases $[2,10]$ ). People catch the infection by contact with affected animals, other people or soil.

Clinically, tinea capitis is characterized by one to a few irregularly shaped, scaling and alopecic patches, in which the hair is broken off at a varying level from the hair follicle [1,2].

Diagnosis is made by clinical examination, observation with microscope and colture [11].

Treatment is based on systemic griseofulvin or terbinafine for at least 8 weeks [10]. 
If not diagnosed and treated properly, tinea capitis may reach epidemic proportion.

\section{Patients and Methods}

We conducted a prospective study in the Dermatology Clinic of the University of Turin (Italy) from January 1997 to December 2012. The study included all patients older than 18, whose diagnosis of tinea capitis was suspected clinically and confirmed by mycological examination. Informed consent of all patients was obtained.

Clinical material was microscopically examined with the use of $20 \% \mathrm{KOH}$ solution (potassium hydroxide and dimethylsulphoxide solution) looking for the presence of hyphae and artroconidia; the samples were also inoculated on Sabouraud agar and Mycosel agar for 15 days.

For each patient we collected information about sex, age, nationality, residence, history of contact with animals or affected people, symptoms and aetiological agent.

\section{Results}

In the period from January 1997 to December 2012 we collected 508 cases of tinea capitis, and 13 of these patients were adults. The relative frequency was estimated to be $2.6 \%$ of all cases. Most of the patients were females, with a $\mathrm{M} / \mathrm{F}$ ratio of 0.4 (4 males and 9 females).

Median age at diagnosis was 56.5 years, ranging from 20 to 80 years. Only one patient came from a non-European country (Congo). 7 patients out of 13 lived in rural areas.

All patients were in good immunity conditions, except one who suffered of diabetes and two that were in chronic oral corticosteroid therapy.

Contact with animals was reported in 6 cases (4 cats and 2 rabbits), whereas in one patient there has been a contact with an affected family member (son).

The most frequent aetiological agent was M. canis ( 7 patients, 53.8\%), followed by T. mentagrophytes (3 patients, 23.1\%), T. rubrum (2 patients, $15.4 \%$ ) and $T$. violaceum (1 patient, $7.7 \%$ ).

Clinically 5 patients out of 13 presented a single patch on their head; physical examination showed in 5 cases other localizations of dermatophytoses, in particular we observed an association with tinea corporis, tinea manuum and tinea faciei. In one case the patient displayed lesions on the whole body (T. rubrum Syndrome [12]) 11 patients suffered from itch (Table 1).

Treatment consisted in griseofulvin at the dose of 500 $\mathrm{mg} / \mathrm{d}$ or terbinafine $250 \mathrm{mg} / \mathrm{d}$, accompanied by topical imidazoles for at least 8 weeks.

Partial cicatricial alopecia was seen in 3 patients but most of them recovered completely without any sequela.

\section{Discussion}

Tinea capitis is common in pre-pubertal children, and it is very rare in adults $[3,5]$. We found a relative frequency of $2.6 \%$; this data agrees with literature [2,4], even if there are reported cases of a higher prevalence [13]. The

Table 1. Characteristics of our patients with tinea capitis.

\begin{tabular}{|c|c|c|c|c|c|c|c|c|c|}
\hline $\mathrm{N}$ & Age & Sex & Nationality & Residence & Other Ringworms & Animals Contact & Other Cases in Family & Predisposing Factors & Agents \\
\hline 1) & 29 & $\mathrm{M}$ & I & $\mathrm{R}$ & No & Rabbits & No & No & T. M. \\
\hline 2) & 47 & $\mathrm{~F}$ & I & $\mathrm{R}$ & No & No & No & No & T. M. \\
\hline 3) & 20 & $\mathrm{~F}$ & I & M & No & Cats & No & No & M. C. \\
\hline 4) & 74 & $\mathrm{~F}$ & I & M & Yes & No & No & Ormonal Disfunction & T. R. \\
\hline 5) & 79 & $\mathrm{~F}$ & I & M & No & Cats & No & Diabetes & M. C. \\
\hline 6$)$ & 49 & M & I & $\mathrm{R}$ & Yes & No & Yes & No & M. C. \\
\hline 7) & 65 & $\mathrm{~F}$ & I & $\mathrm{R}$ & Yes & Rabbits & No & No & T. M. \\
\hline 8) & 56 & $\mathrm{~F}$ & I & $\mathrm{R}$ & No & Cats & No & Eczema & M. C. \\
\hline 9) & 35 & M & $\mathrm{C}$ & M & No & No & No & No & M. C. \\
\hline 10) & 67 & $\mathrm{~F}$ & I & M & Yes & No & No & Corticosteroid Therapy & T. R. \\
\hline 11) & 68 & $\mathrm{~F}$ & I & $\mathrm{R}$ & Yes & Cats & No & Collagenous Disease & M. C. \\
\hline 12) & 65 & $\mathrm{~F}$ & I & $\mathrm{R}$ & No & No & No & Corticosteroid Therapy & M. C. \\
\hline 13) & 80 & $\mathrm{~F}$ & I & M & No & No & No & No & T. V. \\
\hline
\end{tabular}

M = Male; F = Female; I = Italian; C = Congolese; $\mathrm{R}$ = Rural Area; $\mathrm{M}=$ Metropolitan Area; $\mathrm{T} . \mathrm{M}$. = Tricophyton mentagrophytes; . $\mathrm{R} .=$ Tricophyton rubrum; M. C. = Microsporum canis; T. V. = Tricophyton violaceum. 
relative resistance of adult's hair to colonization by dermatophytes, probably due to the fungistatic properties of the long chains fatty acids of the sebum produced after puberty and unknown immunological factors, could explain the rareness of cases in the elderly $[2,4,6]$. While literature says that tinea capitis is more common in little boys [6], in our study we saw a female prevalence of the disease, in particular in post-menopausal women; this is thought to be related to increased involution of sebaceous glands following decreased blood estrogen level $[1,2,6]$; furthermore taking care of children and more frequent visits to the hairdresser could help to explain this higher incidence $[2,14,15]$.

Another important factor predisposing to tinea capitis is impairment of health or immunity $[2,4,10,11,16]$ : in fact, one of our patient suffered from diabetes and two were in oral chronic corticosteroid therapy.

According to literature, $M$. canis was found to be the most frequent aetiological agent in our report $[3,5,6,16]$.

Tinea capitis caused by infection of $T$. rubrum is described to be extremely rare [4,17]; however we observed two patients affected by this pathogen, and one of them suffered from $T$. rubrum syndrome (skin ringworm all over the body [12]).

\section{Conclusion}

In conclusion, this study wants to highlight the importance of considering tinea capitis as a differential diagnosis in elderly patients with scaly scalp lesions, even though it is considered to be rare in adults.

\section{REFERENCES}

[1] E. Elewski, “Tinea Capitis: A Current Perspective,” Journal of the American Academy of Dermatology, Vol. 42, No. 1, 2000, pp. 1-20. http://dx.doi.org/10.1016/S0190-9622(00)90001-X

[2] A. Mebazaa, K. E. Oumari, N. Ghariani, A. F. Mili, C. Belajouza, R. Nouira, M. Denguezli and M. Ben Said, “Tinea Capitis in Adults in Tunisia," International Journal of Dermatology, Vol. 49, No. 5, 2010, pp. 513-516. http://dx.doi.org/10.1111/j.1365-4632.2010.04296.x

[3] G. Ginter-Hanselmayer, W. Weger, M. Ilkit and J. Smolle, "Epidemiology of Tinea Capitis in Europe: Current State and Changing Patterns,” Mycoses, Vol. 50, No. S2, 2007, pp. 6-13.

http://dx.doi.org/10.1111/j.1439-0507.2007.01424.x

[4] A. Ziemer, K. Kohl and G. Schröder, “Trichophyton Rubrum-Induced Inflammatory Tinea Capitis in a 63-YearOld Man,” Mycoses, Vol. 48, No. 1, 2005, pp. 76-79. http://dx.doi.org/10.1111/j.1439-0507.2004.01069.x

[5] C. Seebacher, J. P. Bouchara and B. Mignon, “Updates on the Epidemiology of Dermatophyte Infections," Mycopathologia, Vol. 166, No. 5-6, 2008, pp. 335-352.

http://dx.doi.org/10.1007/s11046-008-9100-9

[6] C. Gianni, R. Betti, E. Perotta and C. Crosti, "Tinea Capitis in Adults,” Mycoses, Vol. 38, No. 7-8, 1995, pp. 329331.

http://dx.doi.org/10.1111/j.1439-0507.1995.tb00417.x

[7] O. Cervetti, M. Forte and A. Paggio, "Tinea Capitis in Adults: Description of Three Cases,” Minerva Dermatologica, Vol. 125, 1990, pp. 27-28.

[8] C. Seebacher, D. Abeck, J. Brasch, O. Cornely, G. Daeschlein, I. Effendy, G. Ginter-Hanselmayer, N. Haake, G. Hamm, U. C. Hipler, H. Hof, H. C. Korting, A. Kramer, P. Mayser, M. Ruhnke, K. H. Schlacke and H. Tietz, "Tinea Capitis,” Journal der Deutschen Dermatologischen Gesellschaft, Vol. 4, No. 12, 2006, pp. 1085-1091. http://dx.doi.org/10.1111/j.1610-0387.2006.06133.x

[9] T. Hillary and E. Suys, "An Outbreak of Tinea Capitis in Elderly Patients,” International Journal of Dermatology, 2013. http://dx.doi.org/10.1111/j.1365-4632.2012.05817.x

[10] N. Lateur, J. André, J. De Maubeuge, M. Poncin and M. Song, "Tinea Capitis in Two Black African Adults with HIV Infection,” British Journal of Dermatology, Vol. 140, No. 4, 1999, pp. 722-724.

http://dx.doi.org/10.1046/j.1365-2133.1999.02778.x

[11] K. Narang, M. Pahwa and V. Ramesh, "Tinea Capitis in the Form of Concentric Rings in an HIV Positive Adult on Antiretroviral Treatment," Indian Journal of Dermatology, Vol. 57, No. 4, 2012, pp. 288-290. http://dx.doi.org/10.4103/0019-5154.97672

[12] L. Piñeiro, J. Larruskain, P. Idigoras and E. Pérez-Trallero, "Trichophyton rubrum Syndrome: The Tip of the Iceberg and a Preventable Outcome,” Mycoses, Vol. 53, No. 2, 2010, p. 186. http://dx.doi.org/10.1111/j.1439-0507.2008.01685.x

[13] G. Cremer, I. Bournerias, E. Vandemeleubroucke, R. Houin and J. Revuz, "Tinea Capitis in Adults: Misdiagnosis or Reappearance?” Dermatology, Vol. 194, No. 1, 1997, pp. 8-11. http://dx.doi.org/10.1159/000246048

[14] A. T. Vidimos, C. Camisa and K. J. Tomecki, "Tinea Capitis in Three Adults," International Journal of Dermatology, Vol. 30, No. 3, 1991, pp. 206-208. http://dx.doi.org/10.1111/j.1365-4362.1991.tb03853.x

[15] A. Takwale, S. Agarwal, S. C. Holmes and J. Berth-Jones, "Tinea Capitis in Two Elderly Women: Transmission at the Hairdresser," British Journal of Dermatology, Vol. 144, No. 4, 2001, pp. 898-900. http://dx.doi.org/10.1046/j.1365-2133.2001.04154.x

[16] N. Aste, M. Pau and P. Biggio, "Tinea Capitis in Adults," Mycoses, Vol. 39, No. 7-8, 1996, pp. 299-301. http://dx.doi.org/10.1111/j.1439-0507.1996.tb00142.x

[17] H. Bargman, J. Kane, M. L. Baxter and R. C. Summerbell, "Tinea Capitis due to Trichophyton rubrum in Adult Women,” Mycoses, Vol. 38, No. 5-6, 1995, pp. 231-234. 